INSTRUCTIONS AUX AUTRICES/AUTEURS

\title{
PRÉSENTATION DE LA REVUe
}

ASDIWAL. Revue genevoise d'anthropologie et d'histoire des religions est une publication scientifique avec comité de lecture. Tous les textes proposés seront soumis à l'évaluation du Comité scientifique.

Tous les textes proposés à la rédaction seront soumis à une double évaluation anonyme par des pairs (double blind peer review).

Les propositions peuvent être envoyées sous format électronique .doc ou .docx à l'adresse :

$$
\text { info@asdiwal.ch. }
$$

$A S D I W A L$ est une revue francophone qui met l'accent sur les publications en français. Nous accueillons toutefois également les propositions en anglais, allemand ou italien sous réserve de l'approbation du comité de rédaction.

Les auteurs dont la langue de l'article n'est pas leur langue maternelle feront corriger leur texte par une personne qualifiée au préalable.

La revue peut accueillir, dans ses numéros thématiques, des actes de colloque.

\section{EXTRAITS DE LA DÉCLARATION ÉTHIQUE DE LA REVUE}

\section{Art. 1.5. Décision sur la publication}

a. Les articles proposés pour publication par les autrices/auteurs dans les rubriques «Dossiers » et «Études» de la revue sont évalués par le comité de rédaction, qui se laisse guider par la politique éditoriale de la revue, en tenant compte des obligations légales concernant la diffamation, les droits d'auteur et le plagiat. En accord avec le comité scientifique, le comité de rédaction peut refuser un article avant de le soumettre au processus d'évaluation par les pairs à double insu s'il ne suit pas la ligne éditoriale.

b. Sur accord du comité de rédaction, les articles proposés pour publication sont envoyés de manière anonyme pour évaluation à deux relectrices/relecteurs reconnus en tant qu'expert.e.s dans le domaine concerné (évaluation par les pairs à double insu ou double blind peer review). Après un délai maximum de six mois, l'autrice/auteur de l'article proposé pour publication reçoit un rapport de synthèse des deux relectrices/relecteurs et la décision du comité de rédaction: (1) acceptable pour publication; (2) acceptable pour publication après révision ; (3) acceptable pour publication après révision substantielle nécessitant une nouvelle évaluation; (4) non acceptable pour publication. La rédaction peut partager sa décision avec les membres du comité scientifique ou avec les relectrices/relecteurs. Dans le cas d'un appel de la décision, la rédaction peut solliciter de nouveaux relectrices/relecteurs. 
c. Les contributions publiées dans les rubriques «Entretiens », « Notules », «Recherche» et «Comptes rendus» sont des articles commandés à leur autrice/auteur par le comité de rédaction. Ils ne sont pas soumis au processus d'évaluation par les pairs à double insu.

\section{Art. 1.6. Détection de plagiat}

Le comité de rédaction lutte contre les infractions à l'éthique de la recherche et ne tolère pas le plagiat. Il examine systématiquement les contributions proposées pour détecter le plagiat, si nécessaire à l'aide de logiciels tels que Compilatio, mis à disposition par l'Université de Genève (https://cataloguesi.unige.ch/compilatio).

\section{Art. 1.7. Corrections et retraits d'articles}

Si cela s'avère nécessaire pour un article, le comité de rédaction prendra en considération la publication de correctifs ou de clarifications, le retrait de l'article ou la publication d'excuses, selon les directives formulées par le COPE (directives 2019: https://publicationethics.org/retractionguidelines).

\section{Art. 1.8. Politique de traitement des données (Data policy) et archivage.}

a. Un an après sa parution, l'intégralité de chaque numéro de la revue Asdiwal est publié en ligne en accès libre sur le portail Persée, sous la licence CC BY-NC-SA.

b. Les autrices/auteurs disposent en tout temps du droit à publier des articles basés sur leurs données de recherche, y compris après avoir publié un article dans la revue.

c. La politique concernant les métadonnées OAI-PMH (Open Archives Initiative Protocole for Metadata Harvesting) de la revue est celle du portail Persée.

d. L'archivage en ligne pérenne de tous les anciens numéros de la revue (excepté le numéro courant) est assuré par le portail Persée.

\section{Obligations des autrices/auteurs}

\section{Art. 3.1. Validité des informations}

Les informations contenues dans les manuscrits soumis pour publication doivent présenter les résultats des recherches effectuées par les autrices/auteurs ainsi qu'une discussion objective de ces résultats et leur importance. Les données sous-jacentes doivent être présentées correctement. Les informations frauduleuses et consciemment imprécises sont considérées comme des actes contraires à l'éthique et ne sont pas acceptables.

L'identification des recherches faites par les autres doit toujours être clairement donnée. Les autrices/auteurs doivent citer les publications qui ont influencé l'étude en question.

\section{Art. 3.2. Originalité et plagiat}

Les autrices/auteurs doivent garantir d'avoir écrit une étude complètement originale, et s'ils/elles ont utilisé des ouvrages ou des propos d'autres personnes, ceux-ci doivent être correctement cités. 


\section{Art. 3.3. Publications multiples}

Un.e autrice/auteur ne doit pas soumettre des manuscrits représentant la même étude à plus d'une revue (ou ouvrage). La soumission du même manuscrit en parallèle dans plus d'une revue est contraire à l'éthique et n'est pas acceptable.

ASDIWAL accepte les articles publiés originellement dans d'autres langues que la langue de l'article français. Dans ces cas, les autrices/auteurs doivent donner la référence de la première publication et s'assurer qu'elle soit libre des droits d'auteur de l'éditeur original.

\section{Art. 3.4. Paternité du manuscrit}

$\mathrm{Ne}$ sont considérés comme autrices/auteurs que ceux qui ont contribué significativement à la réalisation de l'étude en question. Tous ceux qui ont contribué à l'étude doivent être présents dans la liste des autrices/auteurs. Si d'autres personnes ont participé à certains aspects du projet de recherche, elles doivent être mentionnées dans les remerciements.

L'autrice/auteur principal.e doit garantir que tous les co-autrices/co-auteurs et seulement eux sont bien inclus dans la liste des autrices/auteurs du manuscrit, que les co-autrices/co-auteurs ont vu et approuvé la version finale du manuscrit, et qu'ils ont donné leur accord à la soumission du manuscrit.

\section{Art. 3.5. Révélation de l'information et conflits d'intérêts}

Toutes et tous les autrices/auteurs doivent indiquer, à la suite de leur présentation biographique, les conflits d'intérêts qui pourraient avoir une incidence sur leur projet de publication. Les financements des projets de recherche ayant rendu possible l'étude doivent être indiqués.

\section{Art. 3.6. Erreurs dans la publication}

Si l'autrice/auteur découvre une erreur importante ou une inexactitude dans sa publication, son obligation est d'en informer rapidement la rédaction et d'envisager, en accord avec la/le responsable, le retrait de l'article ou la publication de l'information concernant l'erreur. 


\section{GÉNÉRALITÉS}

\section{Études}

Les études proposées à la revue ne dépasseront pas 50’000 signes (notes et espaces compris). Chaque article sera accompagné :

- d'un résumé en français et en anglais (800 signes, espaces compris)

- de cinq mots-clés en français et en anglais

- de l'indication de l'établissement de rattachement de l'auteur.e et son adresse postale

- du courriel de l'auteur.e

Les auteurs veilleront à mettre leur contribution aux normes éditoriales de la revue.

Les auteurs seront informés qu'ils recevront une réponse environ six mois après la soumission du texte.

Délai de soumission : 31 mars de l'année courante

\section{Comptes rendus}

Les comptes rendus ne dépasseront pas les 8’000 signes (espaces compris). La présentation des volumes suivra le modèle suivant :

Jonathan Z. SMith, On Teaching Religion, Christopher Lehrich éd., Oxford, Oxford University Press, 2013, viii +164 pages, ISBN.

Les citations trop longues des textes recensés sont à éviter.

Dans la mesure du possible, on évitera les notes de bas de page.

Délai de soumission : $1^{\text {er }}$ juillet de l'année courante

\section{INDICATIONS TYPOGRAPHIQUES}

\section{Structure des manuscrits}

Le titre de la contribution apparaîtra en minuscule, police «Times New Roman », 12 points, en gras et centré. À la ligne suivante, l'auteur indiquera son nom complet et le nom de l'institution à laquelle il est rattaché.

Pour le corps de texte, l'auteur emploiera également la police «Times New Roman », 12 points, justifié, interligne 1,5 .

Les intertitres seront indiqués en gras pour le premier niveau d'intertitre, en italique pour le second. Ils seront précédés et suivis d'une ligne blanche.

\section{Indications générales}

Les expressions et mots étrangers seront composés en italique. Les citations en langue étrangère seront composées en italique et mises entre guillemets.

Le cas échéant, les mots hébreux, grecs, japonais etc. seront translittérés selon les standards en vigueur. 
Les indications de siècles seront toujours données en chiffres romains en PETITES CAPITALES.

On composera en chiffres romains majuscules (capitales) les numéros d'ordre des souverains.

$$
\text { Ex. : Elisabeth I }{ }^{\text {ère }} \text {, Charles X. }
$$

\section{Notes}

Les appels de note seront produits automatiquement et en continu.

L'appel de note viendra toujours avant la ponctuation, mais après les guillemets dans le cas d'une citation (il est évident que l'appel de note ne fait pas partie de la citation), également dans un article en anglais, en italien ou en allemand.

Ex. : «L'appel de note viendra toujours avant la ponctuation $»^{1}$.

Les notes seront placées en bas de page.

\section{Citations}

Dans les citations, les termes tronqués seront remplacés par des points de suspension entre parenthèses (...) ; les éventuels ajouts seront indiqués [entre crochets].

On utilisera en premier lieu les guillemets français ( «...»), puis les guillemets anglais (“...”) lorsqu'apparaissent des guillemets dans une citation elle-même placée entre guillemets. Les premiers appellent un espace insécable, les seconds non.

Ex. : « On utilisera en premier lieu les "guillemets français" puis les "guillemets anglais" ».

Pour les paragraphes en retrait (par exemple des citations longues), le texte sera encodé en 11 points. Les paragraphes en retrait seront signalés par une marge de $1 \mathrm{~cm}$ à gauche. Ils seront également justifiés. Il est superflu de mettre les citations en retrait entre guillemets, le retrait tenant lieu de guillemets (N.B. : un appel de note de bas de page situé à la fin d'un paragraphe en retrait sera placé après la ponctuation).

Ex. : Pour les paragraphes en retrait (par exemple des citations longues), le texte sera encodé en 11 points. Les paragraphes en retrait seront signalés par une marge de $1 \mathrm{~cm}$ à gauche. ${ }^{1}$

\section{Typographie}

Les majuscules sont toutes accentuées.

On utilisera des apostrophes typographiques (' et non ' ) et des guillemets typographiques (" et non ").

Lorsque l'on a recours à une incise, celle-ci sera mise soit entre parenthèses, soit entre des tirets (tirets demi-cadratins - et non des traits d'union -).

À l'intérieur de parenthèses, l'on aura recours à des crochets et non à de nouvelles parenthèses. 


\section{Chiffres et nombres}

Les nombres de zéro à seize sont écrits en lettres, à partir de 17 en chiffres. Ils sont tous rédigés en chiffres lorsqu'ils sont mis en relation avec des nombres au-delà de seize.

Ex.: Il s'agit de dieux spécifiques implantés dans différents endroits uniquement dans les provinces centrales : 24 en Yamashiro, 36 en Yamato, 6 en Kawachi, 1 en Izumi, 18 en Settsu.

Tout nombre en début de titre ou phrase est exprimé en lettres.

Ex. : Douze des 22 sanctuaires sont aujourd'hui détruits.

Les chiffres de plus de mille s'écrivent avec des espaces insécables entre les groupes de 3 chiffres : 2934000 .

\section{IMAGES}

ASDIWAL est soucieux d'accorder une place importante dans ses pages aux sources iconographiques. Lorsque les questions abordées par celui-ci le permettent, nous publions une image pleine page en regard du titre de chaque article. Les images apparaissent soit en fin d'article sous forme de cahier iconographique, soit dans le texte, en fonction du choix de l'auteur ou des solutions graphiques.

ASDIWAL publie de préférence des images prises dans leur contexte.

Les renvois éventuels aux images insérées dans le corps du texte seront indiqués sous le format (Fig. 1), entre parenthèses et en gras.

L'auteur devra fournir :

- soit des reproductions photographiques de qualité professionnelle fournies par des institutions (banque d'images de la BNF par exemple).

- soit les références complètes des images afin que l'équipe rédactionnelle puisse, le cas échéant, les commander pour publication scientifique.

- soit le document à reproduire lui-même, s'il est en possession de l'auteur.

- soit tout document photographique (réalisé par l'auteur ou non) s'il s'agit de bâtiments, costumes, gestes, etc.

- soit des scans d'images réalisés avec un scanner photo, en mode photo, couleur (profil rvb98), 600 dpi minium, au format .psd, .tiff, ou .jpg. Le graphiste se chargera du traitement colorimétrique des fichiers.

L'auteur devra également fournir une liste des images, avec une légende précise (format, support, provenance etc.) et, le cas échéant, les crédits photographiques ou autres. Les images et la liste légendée seront transmises dans des fichiers distincts de l'article proprement dit. 


\section{RÉFÉRENCES BIBLIOGRAPHIQUES}

\section{Abréviations usuelles}

Les nom et prénom des auteurs d'une référence bibliographique seront écris en toutes lettres.

Dans les collectifs, l'éditeur (les éditeurs) ou (les) directeur(s) de publication sont (seront) abrégé(s) par éd. au singulier et éds. au pluriel.

L'indication de page(s) sera abrégée par p. s'il s'agit d'un renvoi à une seule page ou pp. s'il s'agit d'un renvoi à plusieurs pages suivi(s) d'un espace insécable.

On prendra soin de distinguer entre suivant(e), abrégé par s., et suivant(e)s, abrégé par ss.

Les références empruntées à une source internet seront mises entre crochets

$$
\text { Ex. : [http ://www.indios.info/] }
$$

\section{Sources}

Nom de l'auteur en PETITES CAPITALES (avec majuscules initiales), Titre de l'œuvre en italique suivi du chant ou livre en chiffres romains, le reste en chiffres arabes. Les numérotations seront séparées par une virgule, qui ne sera pas suivie par un espace.

\section{Ex. : CICÉRON, La nature des dieux II,4.}

L'auteur indiquera, le cas échéant l'édition et/ou la traduction utilisée. Les traducteurs seront indiqués comme suit: PrÉnOM(S) NOM en PETITES CAPITALES, Lieu d'édition, Maison d'édition, année(s) d'édition.

Ex. : EUSĖBE DE CÉSARÉE, Préparation évangélique IX,27,3; traduction d'ÉDOUARD DES PlaCES, Paris, Cerf, 1991.

Les éditions seront indiquées entre parenthèses après la référence. Le nom de l'éditeur, précédé de la mention éd., sera donné EN PETITES CAPITALES (avec majuscules initiales).

$$
\text { Ex. : CALlimaQue, fr. } 34 \text { (éd. Pfeiffer). }
$$

Pour les citations bibliques, le nom du livre sera écrit en entier. Le chapitre et le verset seront séparés par une virgule qui n'est pas suivie par un espace et ils seront indiqués en chiffres romains.

Genèse 24,25 renvoie à Genèse, chapitre 24, verset 25 .

Genèse 24,28-32 renvoie à Genèse, chapitre 24, versets 28 à 32 .

Genèse 24,25.32 renvoie à Genèse, chapitre 24, versets 25 et 32 .

Genèse 29-32 (avec un tiret demi-cadratin) renvoie aux chapitres 29, 30, 31 et 32 de la Genèse. Esaïe 8,23-9,6 renvoie à Esaïe depuis le verset 23 du chapitre 8 jusqu'au verset 6 du chapitre 9 . 
Les textes pseudépigraphiques ou apocryphes seront indiqués en entier et en italique. La numérotation des livres, chapitres, lignes etc. sera donnée en chiffres romains. Comme pour les sources classiques, l'auteur indiquera l'édition et/ou la traduction utilisée.

Ex. : Jubilés 8,12 ; traduction d'ANDRÉ CAQUOT, Paris, Gallimard, 1987.

Pour les sources épigraphiques, papyrologiques ou autres l'auteur emploiera les abréviations courantes, dont il pourra éventuellement fournir une liste dans une note préliminaire.

\section{Publications indépendantes}

PRÉNOM(S) NOM en PETITES CAPITALES (avec majuscules initiales), Titre et sous-titre en italique, nombre de vol., Lieu d'édition en langue originale, Maison d'édition, année(s) d'édition.

Ex. : PhilipPe Borgeaud, Aux origines de l'histoire des religions, Paris, Seuil, 2004.

S'il s'agit d'une réédition, on signalera entre parenthèses, après l'année d'édition, l'année d'édition originale, précédée de l'abréviation orig.

Ex. : Henri JeAnMaire, Histoire du culte de Bacchus, Paris, Payot, 1991 (orig. 1951).

S'il s'agit d'une traduction, on indiquera entre parenthèses, après l'année d'édition, les références à l'édition originale.

Ex. : JAN ASSMANN, Moïse l'Égyptien. Un essai d'histoire de la mémoire, Paris, Aubier, 2001 (Moses the Egyptian. The Memory of Egypt in Western Monotheism, Cambridge, MA, Harvard University Press, 1997).

\section{Contributions à des ouvrages collectifs}

PRÉNOM(S) NOM en PETITES CAPITALES (avec majuscules initiales), "Titre de la contribution », in PRÉNOM(S) NOM(S) éd(s)., Titre et sous-titre du collectif en italique, Lieu d'édition en langue originale, Maison d'édition, année d'édition, pages.

Ex. : YouRI VolokHINE, «Des Séthiens aux impurs. Un parcours dans l'idéologie égyptienne de l'exclusion », in PhilipPe BORgeaud, ThOMAS RÖMER, YOURI VOLOKHINE éds., Interprétations de Moïse. Égypte, Judée, Grèce et Rome, Leiden, Brill, 2009, pp. 199-244.

\section{Articles de revues}

PRÉNOM(S) NOM en PETITES CAPITALES (avec majuscules initiales), « Titre de la contribution », Titre de la revue en italique tomaison ou numéro de la revue en chiffres arabes (année), pages.

Ex. : Nicole Belayche, " "Partager la table des dieux". L'empereur Julien et les sacrifices », Revue de l'histoire des religions 218.4 (2001), pp. 457-486.

\section{Articles de dictionnaires ou d'encyclopédies}

PRÉNOM(S) NOM en PETITES CAPITALES (avec majuscules initiales), s.v. «Titre de la contribution », Nom du dictionnaire en italique tomaison (année), pages ou colonnes (abrégées col. ou cols.).

Ex. : ReNATE SCHLESIER, s.v. « Chthonische Götter », Neue Pauly II (1997), cols. 1185-1190. 


\section{Références déjà données}

Lorsque l'on renvoie plusieurs fois à un même titre, on en donnera les références complètes lors de la première occurrence, puis on l'abrégera ainsi

PRÉNOM(S) NOM en PETITES CAPITALES (avec majuscules initiales), Titre de l'ouvrage ou « Titre de l'article », pages.

Si l'on renvoie au même ouvrage à la référence suivante, on indique Ibid.

Si l'on renvoie à plusieurs ouvrages ou/et articles d'un même auteur dans une même référence, on prendra soin de donner son nom complet dans la première référence, puis d'abréger par ID., EAD. ou EID. (en PETITES CAPITALES, avec majuscule initiale). Les références seront séparées par un pointvirgule.

Ex. : GuY G. STROUMSA, La Fin du sacrifice. Les mutations religieuses de l'Antiquité tardive, Paris, Odile Jacob, 2005 ; ID., A New Science. The Discovery of Religion in the Age of Reason, Cambridge, MA, Harvard University Press, 2010.

\section{Indications générales}

Lorsque l'on cite une page précise d'un article ou d'une contribution à un collectif, on indiquera, dans la mesure du possible, la pagination de l'ensemble de l'article, suivie de l'indication de la page d'où est tirée la citation.

Ex. : YOURI VOLOKHINE, «Des Séthiens aux impurs. Un parcours dans l'idéologie égyptienne de l'exclusion", in PHILIPPE BORgeAud, THOMAS RÖMER, YOURI VOlOKHINE éds., Interprétations de Moïse. Égypte, Judée, Grèce et Rome, Leiden, Brill, 2009, pp. 199-244, ici p. 204.

Pour les titres d'ouvrage et d'article de langue anglaise on mettra des capitales aux initiales de chaque mot (substantifs, verbes, adjectifs, adverbes), à l'exception des prépositions, conjonctions, pronoms relatifs et articles.

Ex.: Guy G. Stroumsa, A New Science. The Discovery of Religion in the Age of Reason, Cambridge, MA, Harvard University Press, 2010.

Les lieux et maisons d'édition multiples seront divisés par des tirets (tirets demi-cadratins - ) et non par des traits d'union ( - ).

Ex. : Oleg Grabar, BenJAmin Z. KeDAR éds., Where Heaven and Earth Meet : Jerusalem's Sacred Esplanade, Jerusalem - Austin, TX, Yad Ben-Zvi Press - University of Texas Press, 2009.

\section{NB : L'envoi de ce document ne vaut pas pour acceptation du manuscrit.}

